\title{
Complementary Filter Performance Enhancement through Filter
} Gain

\author{
Dung Duong Quoc ${ }^{1,3^{*}}$, Jinwei Sun ${ }^{1}$, Van Nhu Le ${ }^{2,3}$ and Lei Luo ${ }^{1}$ \\ ${ }^{I}$ Dept. of Automatic Test and Control, Harbin Institute of Technology, Harbin, \\ China \\ ${ }^{2}$ Research center for Space Optics Engineering, Harbin Institute of Technology, \\ China \\ ${ }^{3}$ Le Qui Don Technical University, Cau Giay, Ha Noi, Viet Nam \\ *Corresponding author: Jinwei Sun, Email: jwsun@hit.edu.cn
}

\begin{abstract}
Complementary filters coupled with MEMS IMU are preferred in applications where computational simplicity, low power and low cost is of prime importance. Such algorithms are equipped with fixed filter's gain, however improvements can be realized by changing the filter's gain as per the dynamic situation experienced by the platform. This paper is intended to evaluate the performance of conventional fixed gain complementary algorithm against adoptive gain complementary filter for attitude estimation using MEMS IMU as a test subject. As only IMU (Inertial Measurement Unit) has been exploited without using any aided sensory system, so the mandate is limited to evaluate performance of these algorithms on the basis of Euler angles roll and pitch estimation. Significant performance improvement is observed by varying filter gain in accordance with dynamic situation experienced by the system.
\end{abstract}

Keywords: Attitude Estimation, IMU, Data Fusion, INS

\section{Introduction}

Complementary algorithms and the likes are the results of the decades-long research in the pursuit for computationally simple algorithms. Attitude estimators can be categorically considered computationally simple with excellent performance in most applications. They are efficient, robust and can be applied to complex algorithms as well, however each category has its pros and cons. The computationally simple algorithm's performance, in which complementary filters fall, degrades if some pre-requisite conditions are not met. Complementary filtering searches and explores redundancy of the sensor in order to successfully discard measurement disturbances in complementary frequency regions, without deforming the signal. It is interesting that complementary filters do not consider noise stochastic description which results in slight loss of performance. However it is favorable in the presence of irregular measures that arise out of the expected variance [1]. The second class is computationally expensive solution but so far it is only the de-facto standard for AHRS system. However this category, apart from computational complexity, may not be employed robustly and may exhibit linearity error problems. Kalman filters and the likes fall in this category. The application of these estimators ranges from underwater, aerial and on-surface applications involving UUVs, ROVs, AUVs navigation systems, medical fields, robots and robotic appliances, gaming applications and augmented reality system to industrial control systems [2-4]. The choice of filtering architectures for Inertial Navigation System (INS) can be found in the literature ranging from classical methodologies, to 
lately proposed algorithms [5, 6]. Extended Kalman Filter (EKF) is the most well known and widely used filtering algorithms [6-10], and the Unscented Kalman Filter (UKF) has been there as an alternative to the EKF [6], [11, 12], which numerically approximates the mean and covariance of the state estimate parameterized in Euclidean spaces. Recently, there has been growing interest in the design of nonlinear observers that are tentatively stable and produce explicit regions of attraction [13-18]. So far, a number of complementary filters have been worked out and successfully implemented. Initially, in some limited applications, linear complementary filters have been proposed [9]. Next, the nonlinear complementary filters with fixed gain have been designed and developed for a number of applications [10-14]. Nevertheless, these schemes fail when the system undergoes high dynamics and some pre-requisite conditions like steady state conditions are not satisfied. Complementary filters with switching gain have therefore been developed to alleviate the fixed gain complementary filter's restrictions [15-18].

For attitude estimation problem, both internal and external sensory systems have been in use. For a complete 3-D attitude and orientation estimation, both classes of these sensors are needed. MEMS based INS system is evolving technology having inherent noise issues with the benefits of low cost and low power. With MEMS IMU alone, complementary filters estimates attitude in terms of Euler roll and pitch angles as biases are effectively diminished by accelerometers. However, yaw estimation is not observable to accelerometer. A complete AHRS (Attitude Heading \& Reference System) uses IMU along with Magnetometer or external sensory system such as GPS, APS etc [18-24].

The research presented in this paper is based on miniature sized MEMS MPU 6050. We propose a complementary filter with time varying gain in comparison with the conventional complementary filter having fixed gain. The performance of the two attitude estimators are compared against the accurate but computationally complex Unscented Kalman filter. The paper has been arranged in the following mode. The paper consists of four sections followed by a conclusion. Section 2 presents a general overview regarding complementary algorithm and MEMS IMU. Section 3 provides details of the three filters: the conventional complementary filter (CCF), modified gain complementary filter (MGCF) and unscented Kalman filter (UKF). Section 4 presents simulation result for roll and pitch estimated by the three schemes along with the associated error for each scheme followed by experimental results with MEMS MPU. Finally, the conclusion is provided based on the simulation and experimental results.

\section{Complementary Filter and MEMS IMU Modeling}

\subsection{Complementary Filter}

A Complementary Filter (CF) in the very basic form of filters which falls under the category of computationally less expensive and acceptably accurate sensor fusion method. It can be applied to a number of orientation applications. Both linear and nonlinear implementations of the filters exist and have been extensively researched. For IMU case, estimations based on low frequency response of gyro are not reliable as the bias drift issue makes it impractical. On the other hand, estimations based on low frequency measurements from accelerometer are highly reliable. The reverse is not true for accelerometer. The $\mathrm{CF}$ combines the high-pass filtered estimations from gyro measurements with low pass filtered estimations from accelerometer measurements to alleviate the bias issue as depicted in fig.1. A number of variants of $\mathrm{CF}$ exist, fixed-gain and recently variable/switched gain $\mathrm{CF}$ are being the most significant. 


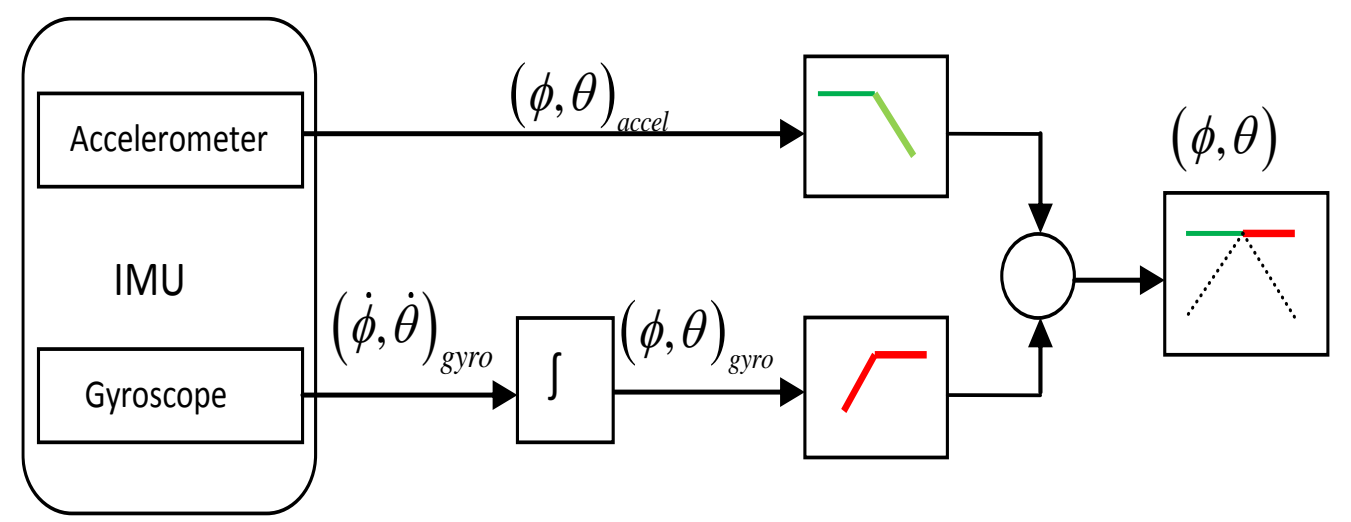

Figure 1. Basic Complementary Filter

\subsection{Attitude Estimation from Gyro}

MEMS IMU is fitted with tri-axial gyros, tri-axial accelerometers and is a miniature size sensor package with the benefits of low cost and low power. Using either gyro or accelerometer alone for attitude estimation does not suffice due to gyro bias and accelerometer inability to distinguish between gravity, rotational and translation acceleration. Sensor fusion algorithms are used to eliminate this problem by either combining the estimations based on reliable low frequency accelerometer output with estimations from high frequency gyro's components (complementary filters methodology) or by de-biasing gyro estimations from accelerometer's components as in Kalman filter approach.

Euler angles roll, pitch and yaw defining the orientation can be determined from triaxis gyros measuring rotation rate. The measurement of MEMS gyro can be modeled as:

$$
\begin{gathered}
\bar{\Omega}_{\text {gyro }}^{b}=\bar{\Omega}^{b}+n_{\Omega}+v_{\Omega} \\
\bar{\Omega}_{\text {gyro }}^{b}=\left[\begin{array}{l}
\omega_{x} \\
\omega_{y} \\
\omega_{z}
\end{array}\right]=\bar{\Omega}^{b}+\left[\begin{array}{l}
n_{\omega_{x}}+v_{\omega_{x}} \\
n_{\omega_{y}}+v_{\omega_{y}} \\
n_{\omega_{z}}+v_{\omega_{z}}
\end{array}\right]
\end{gathered}
$$

Where, $n_{\omega}$ and $v_{\omega}$ represent the gyro noise and the associated bias respectively.

The relationship between rate of Euler angle and gyro output is governed by:

$$
\left[\begin{array}{l}
\dot{\phi} \\
\dot{\theta} \\
\dot{\psi}
\end{array}\right]=\left[\begin{array}{ccc}
1 & \sin \phi \tan \theta & \cos \phi \tan \theta \\
0 & \cos \phi & -\sin \phi \\
0 & \sin \phi / \cos \theta & \cos \phi / \cos \theta
\end{array}\right]\left[\begin{array}{c}
\omega_{x} \\
\omega_{y} \\
\omega_{z}
\end{array}\right]
$$

Where, $\phi, \theta, \psi$ represent roll, pitch and yaw in radian respectively.

If the gyroscope were free from bias, simple integration of Eq. 2 would make it possible to estimate attitude from gyroscope alone. However, as shown in Fig.2, the estimates quickly drift in short duration if only gyroscope is used without any additional sensors. 


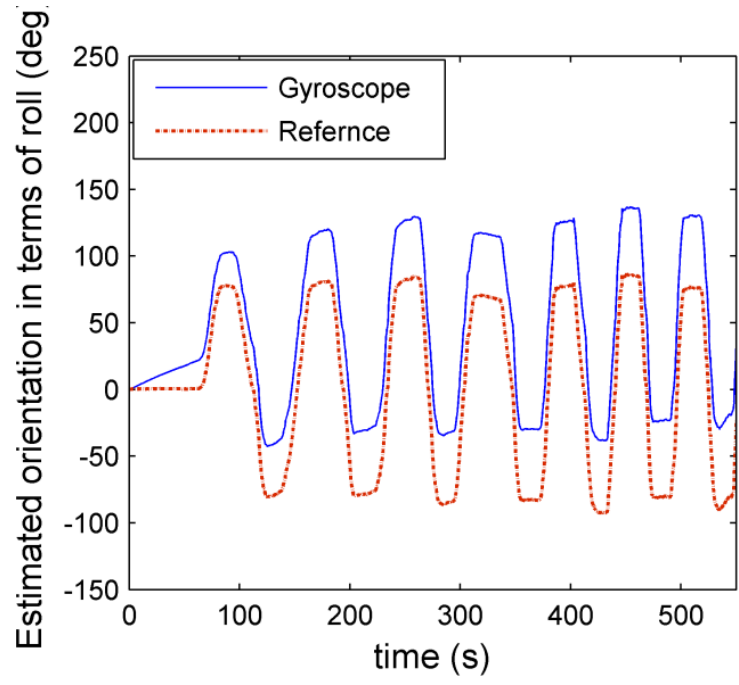

Figure 2. Attitude Estimation based on Gyroscope Data Only

\subsection{Attitude Estimation from Accelerometer}

Accelerometer is used to measure gravity along with rotational and translational acceleration. Euler roll and pitch angles are computed by using accelerometer gravity vector measurement. However, yaw is not observable in this case. Therefore some additional sensory system is used. The tri-Accelerometers measure specific force as folows:

$$
\begin{gathered}
\bar{a}_{\text {accel }}^{b}=\bar{a}+n_{a}+v_{a} \\
\bar{a}_{\text {accel }}^{b}=\left[\begin{array}{l}
a_{x, \text { accel }} \\
a_{y, \text { accel }} \\
a_{z, \text { accel }}
\end{array}\right]=\bar{a}+\left[\begin{array}{l}
n_{a_{x}}+v_{a_{x}} \\
n_{a_{y}}+v_{a_{y}} \\
n_{a_{z}}+v_{a_{z}}
\end{array}\right]
\end{gathered}
$$

Here, $n_{a}$ and $v_{a}$ represent noise and bias of accelerometer respectively. Bias in accelerometer case is negligibly small. The specific force measured by accelerometers constitutes linear/translational, centripetal/rotational acceleration along with gravity. MEMS tri-axial accelerometer measuring gravity, linear and rotational acceleration can be modeled as:

$$
\bar{a}^{b}=\dot{\bar{V}}^{b}+\bar{\Omega}^{b} \times \bar{V}^{b}-\bar{g}^{b}
$$

Here, $\dot{\bar{V}}^{b}$ is translational acceleration w.r.t body coordinates, $\bar{\Omega}^{b} \times \bar{V}^{b}$ is rotational acceleration and $\bar{g}^{b}$ is gravity in body coordinates. The problem with accelerometer is; it cannot distinguish between these vectors. In situations where linear and rotational accelerations can be assumed negligible, the specific force measured by accelerometer can be model as:

$$
\left[\begin{array}{l}
a_{x, \text { accel }} \\
a_{y, \text { accel }} \\
a_{z, \text { accel }}
\end{array}\right]=-\bar{g}^{b}=g\left[\begin{array}{c}
\sin \theta \\
-\sin \phi \cos \theta \\
-\cos \phi \cos \theta
\end{array}\right]
$$

Roll and pitch Euler angles can be estimated from accelerometers by employing the fact that the predominant specific force measured by accelerometer is the gravity vector. The roll and pitch estimation based on Eq.5 can be written as: 


$$
\left.\begin{array}{l}
\text { Roll }=\operatorname{inv} \tan \left(\frac{a_{y, a c c e l}}{a_{z, a c c e l}}\right) \\
\text { Pitch }=\operatorname{inv} \tan \left(-\frac{a_{x, a c c e l}}{\sqrt{a_{y, a c c e l}^{2}+a_{z, a c c e l}^{2}}}\right)
\end{array}\right\}
$$

It should be noted that this equation assume zero linear and rotational acceleration and so cannot be employed in situations involving high dynamic. Roll angles estimated from accelerometers data only are depicted in Fig3 using Eq.6. However, as can be observed, the results are very noisy and may not be acceptable in term of accuracy in many situations.

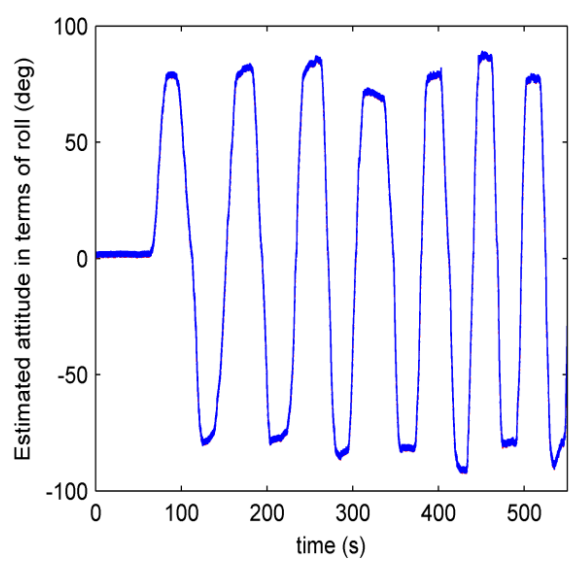

(a)

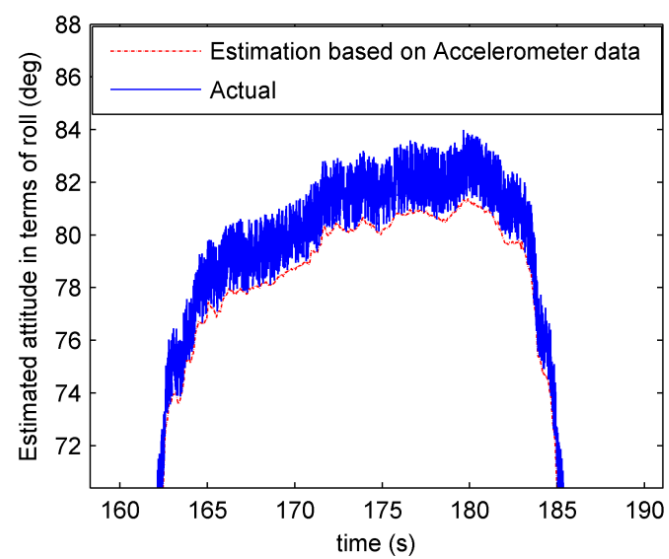

(b)

Figure 3. (a) Attitude Estimation based on Accelerometer Data Only, (b) Zoom-in

A phenomenon called gimbal lock or singularity problem occurs as soon as pitch reaches in vicinity of 90 degrees. Quaternion based algorithms are preferred to avoid this situation.

\section{Attitude Estimating Algorithms}

Orientation estimating algorithms recursively collect inputs from measuring devices/sensors remove the errors and produce a reliable attitude estimate. The measuring sensors may be either internal (IMU etc) or external or aided (GPS etc) whereas the data fusion algorithm may be computationally simple (complementary filter) or complex (Kalman filter). High accuracy comes with price but there are many applications where the less expensive MEMS sensors and computationally inexpensive algorithms can be employed efficiently.

\subsection{Conventional Complementary Filter $(\mathrm{CCF})$}

As demonstrated by Eq.2 and Eq.6, attitude can be determined from gyro as well as Accelerometers. Where gyro inherits the bias issue (low frequency), accelerometer low frequency response is reliable but not at the high frequencies. All complementary filters, in one form or the other, fuse the two attitude estimation in such a fashion that low pass filtering is performed on accelerometer attitude estimation where as high frequency filtering for gyros there by removing the errors and produce a reliable estimate. The conventional fixed gain complementary filters usually employ quaternion representation, 
measure inertial direction measurement $(\bar{v})$ from accelerometer data and fuses with gyro angular rate $(\bar{\Omega})$ in the following equation:

Where

$$
\hat{\Omega}^{b}=\bar{\Omega}^{b}+K_{p} e+K_{I} \int e
$$

$$
\begin{gathered}
e=\bar{v} \times \hat{v} \\
\dot{\hat{q}}=\frac{1}{2} \hat{q} \otimes p\left(\hat{\Omega}^{b}\right)
\end{gathered}
$$

Where

$$
\begin{aligned}
& p\left(\hat{\Omega}^{b}\right)=\left(0, \hat{\Omega}^{b}\right)=\left[\begin{array}{llll}
0 & \hat{\omega}_{x}^{b} & \hat{\omega}_{y}^{b} & \hat{\omega}_{z}^{b}
\end{array}\right] \\
& \hat{v}=\left[\begin{array}{l}
2\left(\hat{q}_{2} \hat{q}_{4}+\hat{q}_{1} \hat{q}_{3}\right) \\
2\left(\hat{q}_{3} \hat{q}_{4}+\hat{q}_{1} \hat{q}_{2}\right) \\
\hat{q}_{1}^{2}-\hat{q}_{2}^{2}-\hat{q}_{3}^{2}+\hat{q}_{4}^{2}
\end{array}\right]
\end{aligned}
$$

Here, $\bar{\Omega}^{b}$ is the angular velocity given by gyro (in body coordinates), $K_{p}, K_{i}$ are proportional and integral gains respectively-the adjustable and tuning parameters, $\bar{v}$ is the measured inertial direction based on accelerometer data, $\hat{v}$ is the estimated inertial direction, $e$ is the error between the two whereas $\hat{q}$ is the estimated orientation expressed in quaternion and $\otimes$ is a quaternion product operator.

\subsection{Modified Gain Complementary Filter (MGCF)}

Modified gain complementary filter is an extension of conventional fixed gain complementary filter where the filter gain changes according to system dynamics. The proportional and integral gain in term of resonant frequency and damping factor can be written as:

$$
\left.\begin{array}{l}
K_{i}=\omega_{0}^{2} \\
K_{p}=2 \varsigma \omega_{0}
\end{array}\right\}
$$

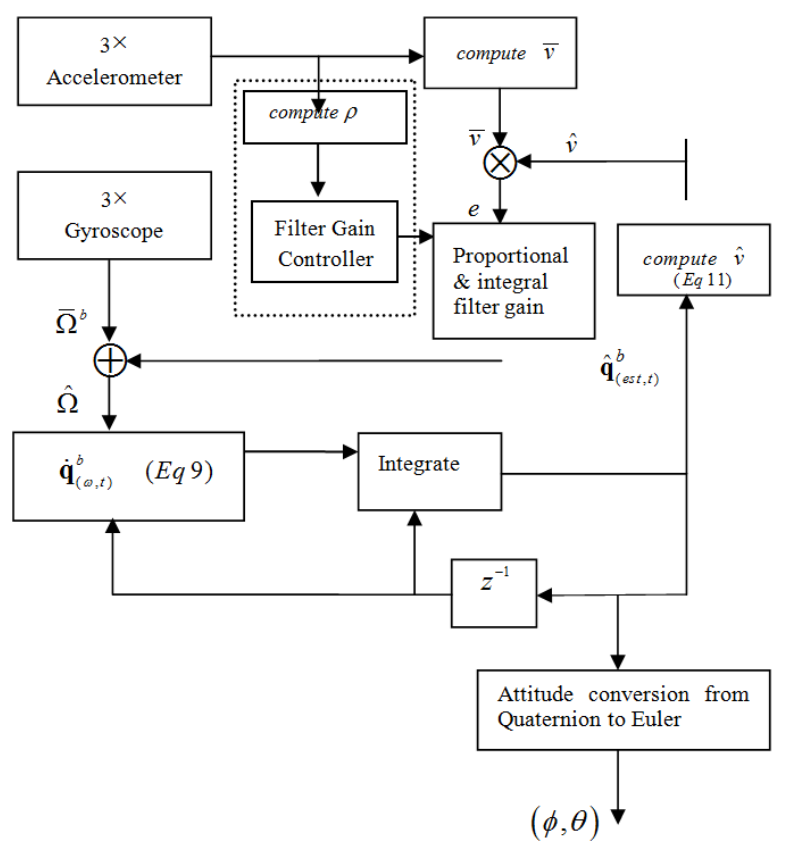

Figure 4. Modified Gain Complementary Filter (MGCF) 
The damping ratio determines the overshoot at cutoff frequency and should be selected such that to ensure smooth frequency response as well as fast bias estimation. Usually, damping ratio $\varsigma \geq 1$ is fixed and so proportional and integral gain can be tuned on by using cutoff frequency. Selecting different cutoff frequencies for different dynamics varies the filter gain and hence enables the filter to cope with different situations. To measure the dynamics, accelerometer and/or gyro measurement can be exploited. A dimensionless parameter $\rho$ can be employed in the following manner:

$$
\left.\begin{array}{c}
\rho(k)=\frac{\left|\sqrt{a_{x}(k)^{2}+a_{y}(k)^{2}+a_{z}(k)^{2}}-g\right|}{g} \\
=\left|\sqrt{a_{x}(k)^{2}+a_{y}(k)^{2}+a_{z}(k)^{2}}-1\right|
\end{array}\right\}
$$

Where $a(k)$ is accelerometer measurement in unit of $g$. In general, the higher the value of $\rho$, the higher is the vibration and dynamic situation and vice versa. The whole idea is summarized in Figure 4.

\subsection{Unscented Kalman Filter (UKF)}

Kalman filter is still the most employed and de facto standard attitude estimator inspite of computational complexity and linearity problem. The states for UKF are defined as:

$$
\bar{x}=\left[\begin{array}{lll}
X_{1} & X_{2} & X_{3}
\end{array}\right]^{T}
$$

Where,

$$
\left.\begin{array}{l}
X_{1}=-\sin \theta \\
X_{2}=\sin \phi \cos \theta \\
X_{3}=\cos \phi \cos \theta
\end{array}\right\}
$$

$\phi$ and $\theta$ are roll and pitch in radian respectively. The process and measurement model can be written as:

$$
\begin{gathered}
{\left[\begin{array}{c}
\dot{X}_{1} \\
\dot{X}_{2} \\
\dot{X}_{3}
\end{array}\right]=\left[\begin{array}{ccc}
0 & \omega_{z} & \omega_{y} \\
-\omega_{z} & 0 & \omega_{x} \\
\omega_{y} & -\omega_{x} & 0
\end{array}\right]\left[\begin{array}{l}
X_{1} \\
X_{2} \\
X_{3}
\end{array}\right]+\bar{w}(t)} \\
\bar{z}=\left[\begin{array}{c}
a_{x, \text { accel }} \\
a_{y, \text { accel }} \\
a_{z, \text { accel }} \\
1
\end{array}\right]=\left[\begin{array}{c}
-g \hat{X}_{1} \\
-g \hat{X}_{2} \\
-g \hat{X}_{3} \\
\hat{X}_{1}^{2}+\hat{X}_{2}^{2}+\hat{X}_{3}^{2}
\end{array}\right]+\bar{r}(t)
\end{gathered}
$$

Where $\omega, \bar{w}(t), a_{\text {accel }}$ and $\bar{r}(t)$ are gyro rotation rate in radian per second, white Gaussian process noise, accelerometer measured specific force in unit of $\mathrm{g}$ and measurement noise respectively. 


\section{Results and Discussion}

\subsection{Simulated Data Results}

Matlab simulated data was used mimicking low and high dynamic situations. Damping factor was fixed at 2 for both CCF and MGCF. Based on Eq. 13, MGCF gain was tuned for different dynamic situation as:

a. Zero dynamics/acceleration: For $\rho(k)<0,01$, zero linear and rotational acceleration assumption per Eq. 5 is fully valid thereby trusting fully on accelerometer measuring gravity; cut off frequency in this case is assumed $0.1 \mathrm{rad} / \mathrm{s}$.

b. Low dynamics: For $0,01<\rho(k)<0,25$, accelerometer estimation is trusted less. To ensure this, cutoff frequency is switched to $0.07 \mathrm{rad} / \mathrm{s}$ (which controls filter gain by Eq. 12)

c. Medium dynamics: Cut off frequency is 0.035 for $0,25<\rho(k)<0,35$.

d. High dynamics: cut off frequency is zero when $\rho(k)>0,35$.

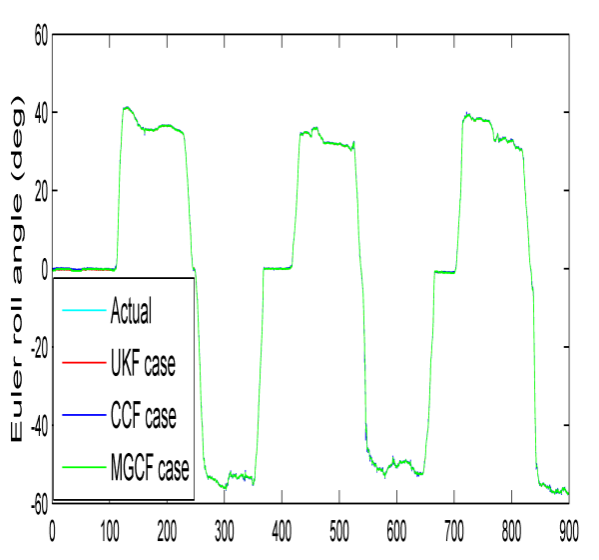

(a)

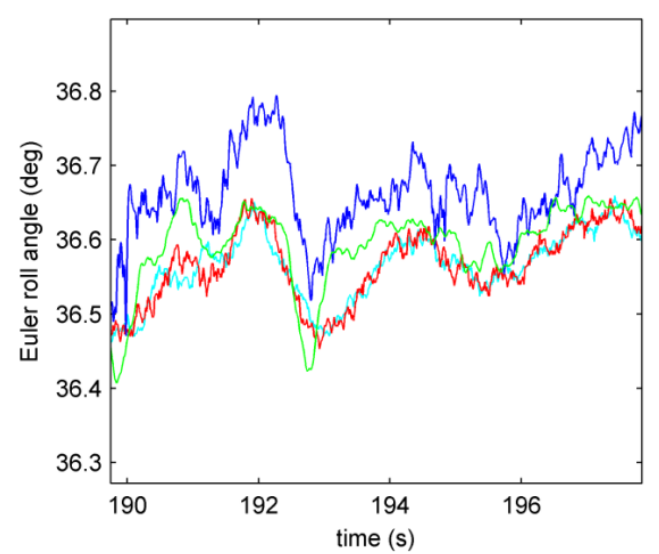

(b)

\section{Figure 5. a. Simulation of Euler Roll Angle Determination UKF, CCF and MGCF, b Close View}

Figures 5, 6 depict low acceleration roll and pitch estimation using CCF, MGCF and UKF in comparison with reference. It is obvious from these results that for a system under dynamic state, MGCF is more accurate in comparison with CCF with the cost of a little computational burden. Table1 summarizes RMSE in roll and pitch estimation for the three estimators which further verify this conclusion. Also, the three algorithms were tested for computational complexity based on execution time. Taking execution time of CCF as one unit, it was observed that MGCF takes 1.37 units and UKF takes 6.95 units which further demonstrate the efficiency of VGCF. 


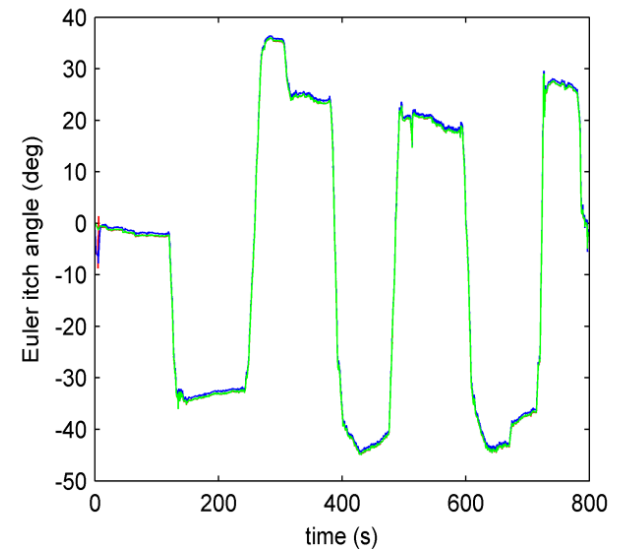

(a)

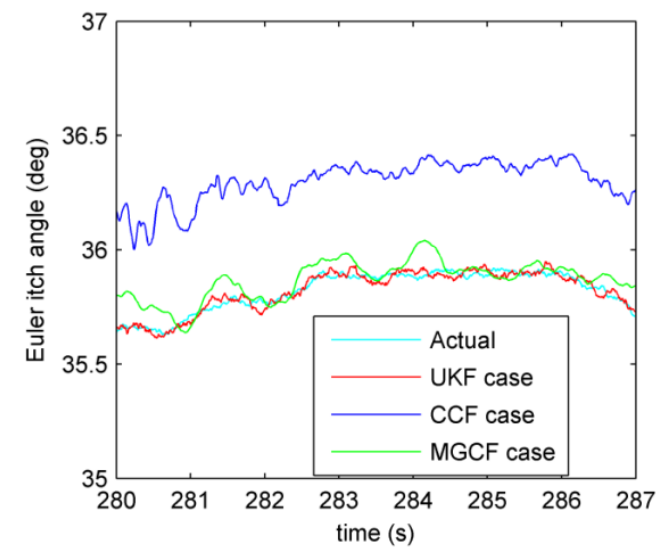

(b)

Figure 6. (a) Simulation of Euler Pitch Angle Determination by UKF, CCF and MGCF, (b) Close View

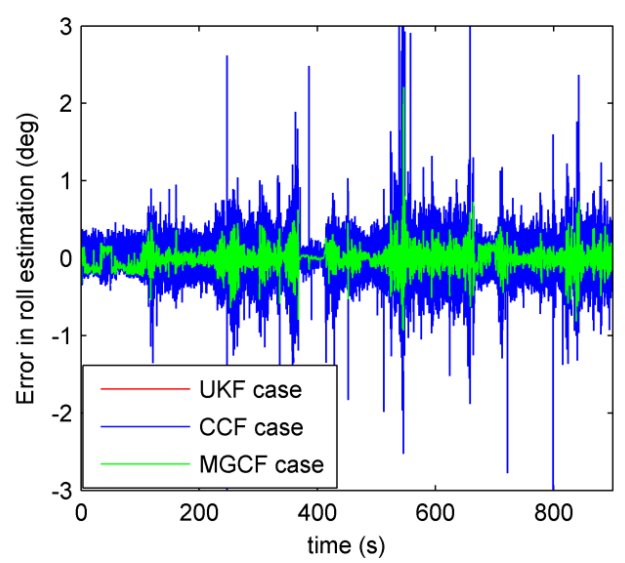

(a)

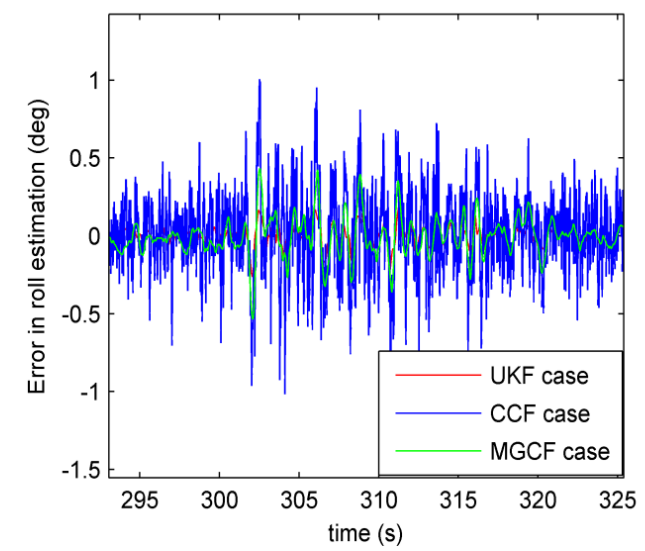

(b)

Figure 7. (a) Error in Roll Determination, (b) Close View

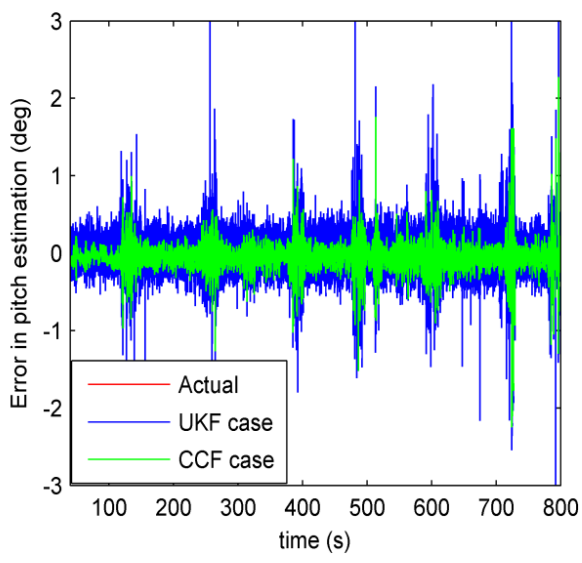

(a)

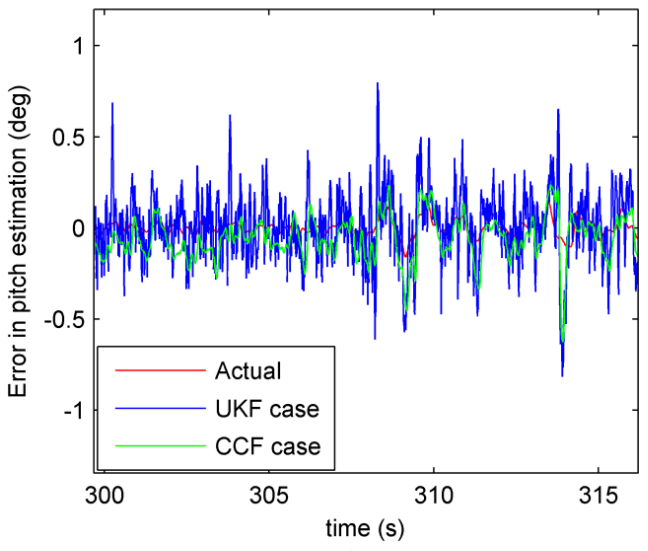

(b)

Fig. 8 (a) Error in Pitch Determination, (b) Close View 
Table 1. Root Mean Square Error (RMSE) for CCF, MGCF and UKF

\begin{tabular}{cccc}
\hline Algorithm & RMSE roll $(\mathrm{deg})$ & RMSE pitch $(\mathrm{deg})$ & Simulated Data \\
\hline CCF & 1.84 & 2.03 & \\
MGCF & 1.07 & 1.32 & Moderate \\
UKF & 0.35 & 0.41 & dynamic system \\
\hline
\end{tabular}

\subsection{Experimental Data Result}

Experiments were carried out using MEMS MPU 6050 by mimicking low, medium and high dynamic situations. The measurements thus obtained were subjected to all the aforementioned algorithms for comparison. It was noted that for system under low and medium dynamics, the model and algorithms perform efficiently accurate; with MGCF closely following UKF. Damping factor was fixed to a value of 2 and cutoff frequency was selected 0.05 for CCF. The MGCF filter gain for low and medium dynamic test was tuned from cutoff frequency as:

a. Cut-off frequency is $0.1 \mathrm{rad} / \mathrm{s}$ for $\rho(k)<0,025$

b. Cut-off frequency is $0.07 \mathrm{rad} / \mathrm{s}$ for $0,025<\rho(k)<0,035$.

c. Cut-off frequency is $0.05 \mathrm{rad} / \mathrm{s}$ for $0,035<\rho(k)<0,05$.

d. Cut-off frequency is 0 for $\rho(k)>0,05$.

Figures 8 and fig 9 depict low and medium dynamics test evaluation where MGCF closely follow UKF in comparison with CCF. Fig 10 shows the dynamic indicator parameter and fig. 11 and fig.12 indicate the filter gain/tuning parameters $\left(K_{p} \& K_{i}\right)$ variations. However, $\mathrm{CCF}$ and $\mathrm{UKF}$ algorithms fail for system under high dynamics for prolong duration (as Eq. 5 is no more valid) whereas MGCF total dependence on gyro results in bias error.

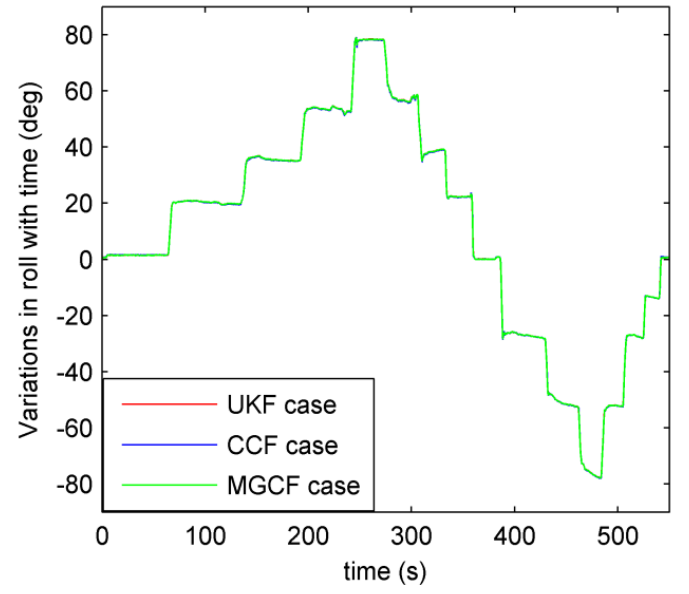

(a)

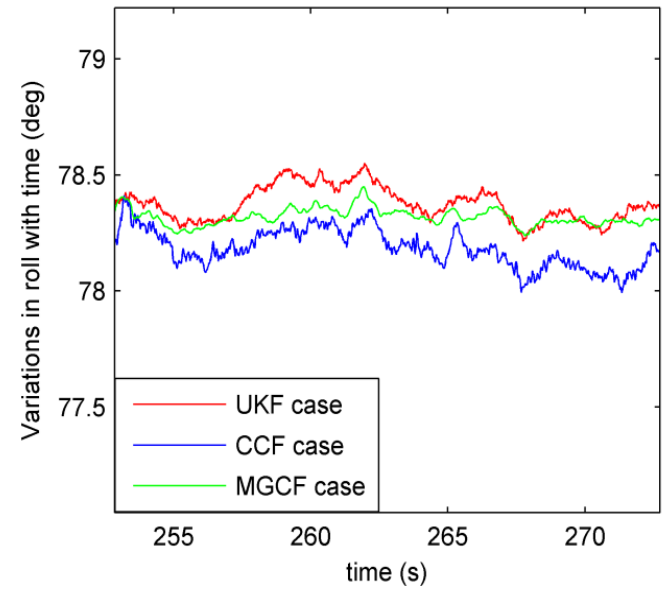

(b)

Figure 8. Roll Angle Determination, (a) Using CCF, MGCF \& UKF, (b) Close View 


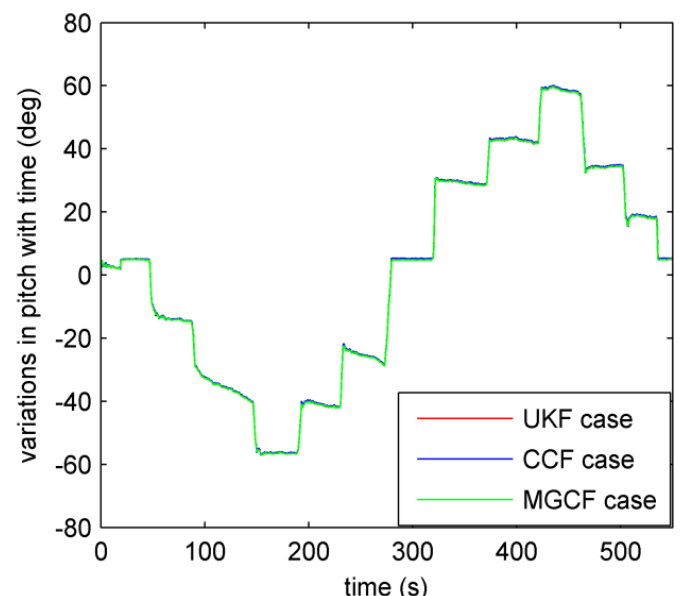

(a)

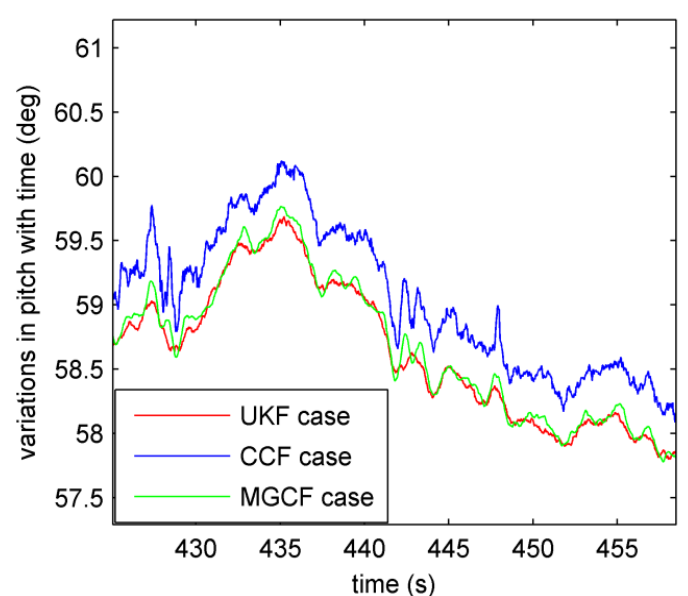

(b)

Figure 9. Pitch Angle Determination, (a) Using CCF, MGCF \& UKF, (b) Close View

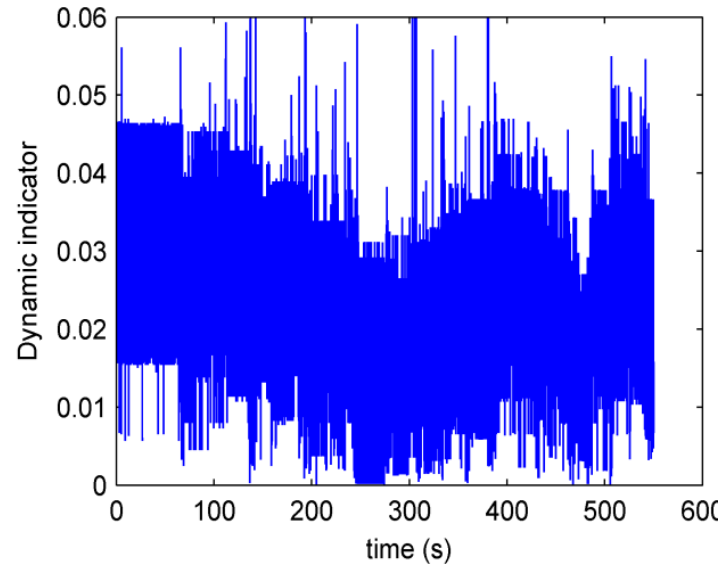

(a)

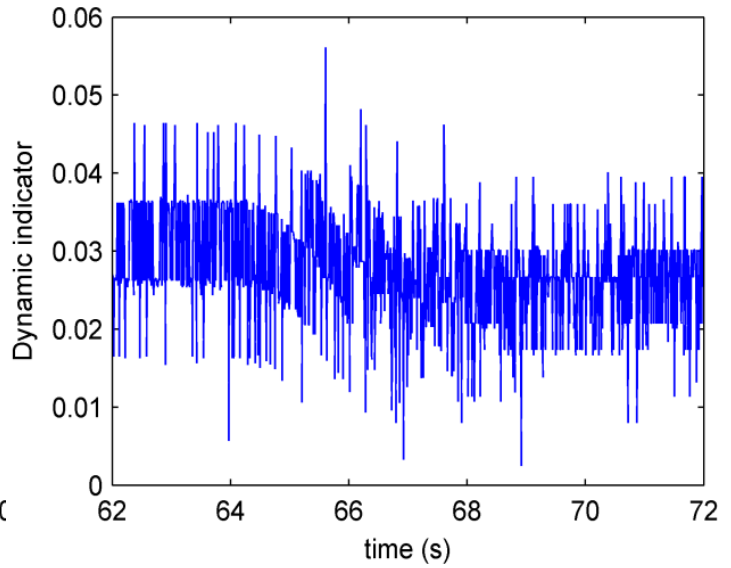

(b)

Figure 10. Dynamic Indicator, $\rho$ (a) variation of $\rho$ during Roll Estimation, (b) Close View

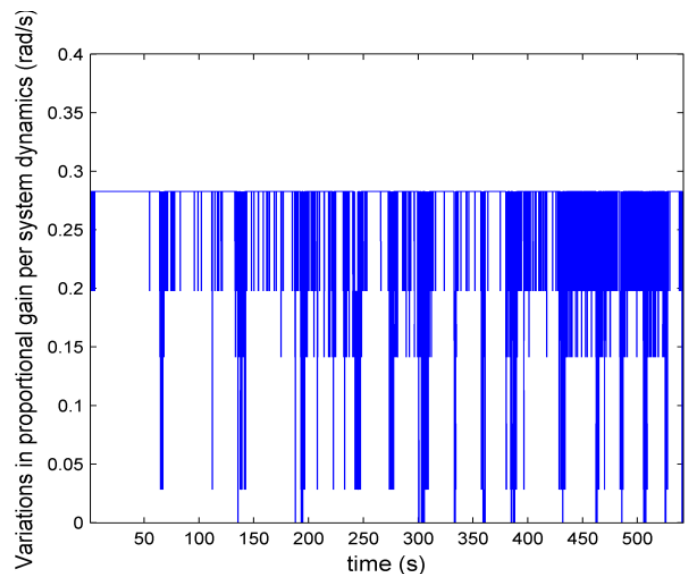

(a)

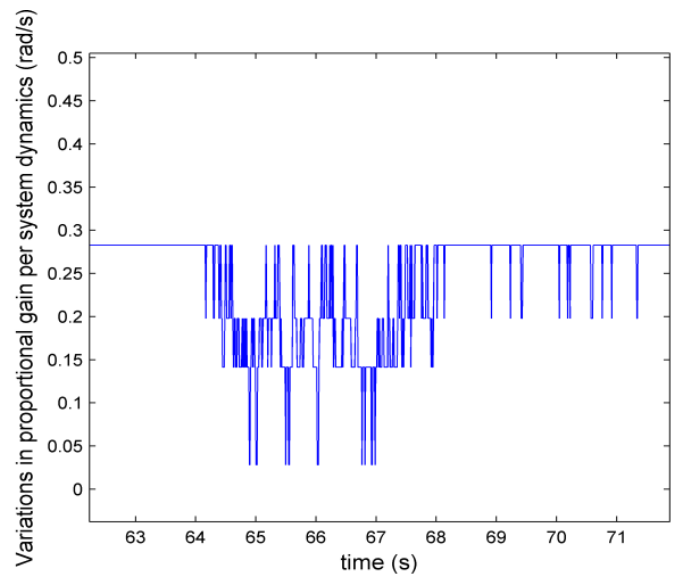

(b)

Figure 11. Variation of Filter Gain per System Dynamics (a) Proportional Gain, (b) Close View 


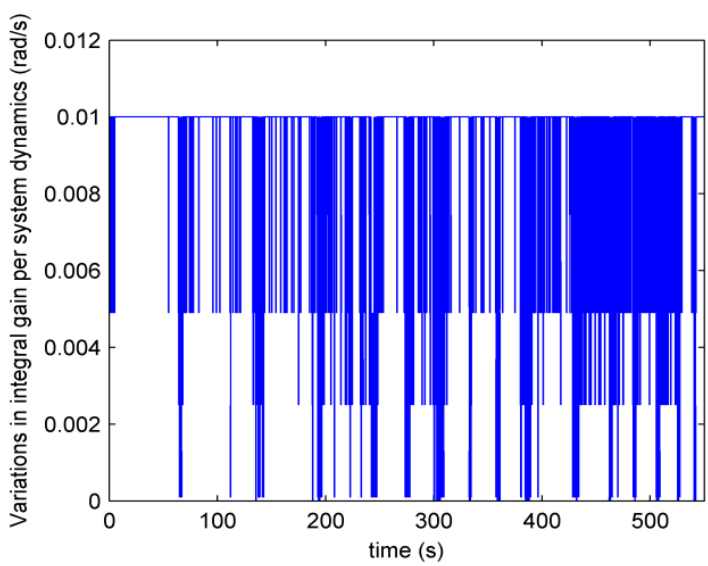

(a)

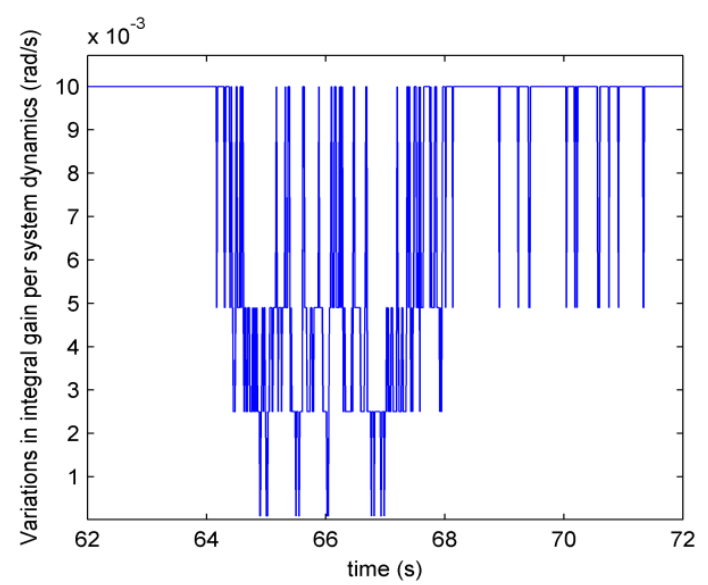

(b)

Figure 12. Variation of Filter Gain per System Dynamics (a) Integral Gain, (b) Close View

\section{Conclusion}

In this research, a low cost, low power, miniature MEMS MPU 6050 was subjected to three types of attitude estimators - CCF, MGCF and UKF; computationally simple, medium and complex in that order. It was observed that roll and pitch Euler angles can be accurately determined from low cost MEMS IMU for system under medium dynamics. In such situation where attitude is to be estimated from IMU only, the accelerometer measuring gravity only assumption results in fairly acceptable accuracy. Moreover, whereas the computationally cheap fix gain complementary filters are efficient solution in many applications, it was demonstrated that accuracy can be improved by varying/switching filter gain with a little computation cost. This is particularly important for applications where computational complexity is of prime concern. For system under high dynamics, estimates from IMU alone are distorted and some aided sensory system is/are to be employed.

\section{References}

[1] R. G. Brown, "Integrated navigation systems and Kalman filtering: A perspective," Journal of the Institute of Navigation, vol. 19, no. 4, (1972), pp. 355-362.

[2] D. Jung and P. Tsiotras, "Inertial attitude and position reference system development for a small UAV," in AIAA Infotech at Aerospace, Rohnert Park, CA, (2007), May.

[3] G. Dissanayake, S. Sukkarieh, and E. Nebot, "The aiding of a low-cost strap-down inertial measurement unit using vehicle model constraints for land vehicle applications," IEEE Transactions on Robotics and Automation, vol. 17, no. 5, (2001) October, pp. 731-747.

[4] P. Batista, C. Silvestre, and P. Oliveira, "Position and velocity navigation filters for marine vehicles," in Proceedings of the 17th IFAC World Congress, Seoul, South Korea, (2008), July.

[5] K. R. Britting, "Inertial Navigation Systems Analysis", John Wiley \& Sons, Inc., (1971).

[6] O. J. Woodman, "An Introduction to Inertial Navigation", (UCAM-CL-TR-696, University of Cambridge, 2007)

[7] D. H. Titterton and J. L. Weston, "Strap down Inertial Navigation Technology (Peter Pegerinus, London, 1997).

[8] R. Munguia and A. Grau, “Attitude and Heading System based on EKF total state configuration”, IEEE Int. Symposium on Industrial Electronics, (2011).

[9] X. Yun, C. Aparicio and E. R. Bachmann, "Implementation and Experimental Results of a QuaternionBased Kalman Filter for Human Body Motion Tracking", Proceedings of IEEE International Conference on Robotics and Automation, (2005), pp. 317-322.

[10] A. J. Baerveldt and R. Klang, "A low cost and low weight attitude estimation system for an autonomous helicopter”, Proceedings IEEE Int. Conf. Intell. Eng. Syst., (1997), pp. 391-395.

[11] J. Roberts, P. Corke, and G. Buskey, "Low-cost flight control system for a small autonomous helicopter," in Proceedings of IEEE Int. Conf. on Robotics and Automation, Taipai, September (2003), pp. 546-551. 
[12] M. Zimmerman and W. Sulzer, "High bandwidth orientation measurement and control based on complementary filtering", the Symp. Robotics Control (SYROCO), Vienna, Austria, (1991).

[13] J. F. Vasconcelos, C. Silvestre, P. Oliveira, P. Batista, and B. Cardeira, "Discrete Time-Varying Attitude Complementary Filter", American Control Conference 2009, (2009), pp. 4056-4061.

[14] S. O. H. Madgwick, A. J. L. Harrison and R. Vaidyanathan, "Estimation of IMU and MARG orientation using a gradient descent algorithm", IEEE International Conference on Rehabilitation Robotics Rehab Week Zurich, ETH Zurich Science City, Switzerland, (2011), June 29 - July 1.

[15] M. Euston and P. Coote, "A complementary filter for attitude estimation of a fixed wing UAV", appeared in IEEE Int Conf on Intelligent Robots and Systems, (2008).

[16] S. Merhav, "Aerospace Sensor Systems and Applications", Springer-Verlag New York Inc., (1998).

[17] C. W. Kang and Park, "Attitude estimation with accelerometers and gyros using fuzzy tuned Kalman filter", Proceed of the European Control Conference, Budapest, Hungary, (2009), August.

[18] T. S. Yoo and S. K. Hong, "Gain-Scheduled Complementary Filter Design for a MEMS Based Attitude and Heading Reference System”, Sensors 2011, 11, 3816-3830; doi: 10.3390/s110403816

[19] M. Wang and Y. C. Yang, "Adaptive filter for a miniature MEMS based attitude and heading reference system", presented in Pro IEEE Pos Loc and Nav Symp, Monterey, CA, USA, (2004) April.

[20] E. Lefferts, F. Markley, and M. Shuster, "Kalman filtering for spacecraft attitude estimation", AIAA J. Guidance, Control, Navig., vol. 5, no. 5, (1982), September, pp. 417-429.

[21] S. J. Julier and J. K. Uhlmann, "Unscented filtering and nonlinear estimation," Proceedings of the IEEE, vol. 92, no. 3, (2004) March, pp. 401-422.

[22] J. L. Crassidis, "Sigma-point Kalman filtering for integrated GPS and inertial navigation," IEEE Transactions on Aerospace and Electronic Systems, vol. 42, no. 2, (2006), pp. 750-756.

[23] X. Kong "INS algorithm using quaternion model for low cost IMU", Robotics and Autonomous Systems, vol. 46, (2004), pp. 221-246.

[24] F. Caron, E. Duflos and D. Pomorski, "GPS/IMU data fusion using multisensor Kalman filtering: introduction of contextual aspects", Information Fusion, vol. 7, (2004), pp. 221230.

\section{Authors}

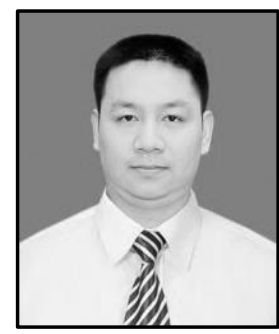

Dung Duong Quoc, he was born in 1981. He received the B.S., M.S. degrees from the Le Qui Don Technical University, Hanoi, Vietnam, in 2007 and 2010, respectively. Now he is pursuing Ph.D. degree in Harbin Institute of Technology (HIT), Harbin, China. His research interests include sensor technology, testing instruments, and signal processing.

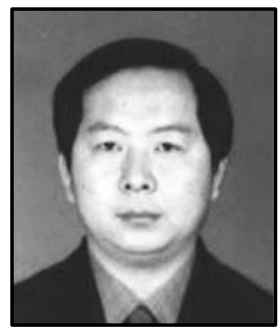

Jin Wei Sun, (M'09) he was born in 1964. He received the M.S. degree in electrical engineering from Harbin Institude of Technology (HIT), Harbin, China, in 1990 and the Ph.D. degree in advanced system control and production from Saga University, Saga, japan, in 2000. He is currently a Professor with HIT. His research interests include sensor technology, testing instruments, and signal processing.

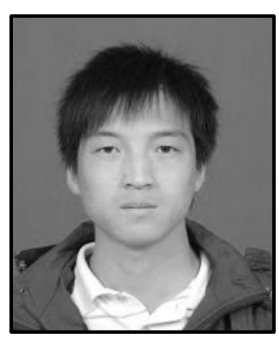

Lei Luo, he was born in 1989. He received the B.S. degree in instrumentation science and Technology from Shenyang University of Technology, Senyang, China, in 2012 and the M.S. degree also in instrumentation science and Technology from Harbin Institude of Technology (HIT), Harbin, China, in 2014. Now he is pursuing the $\mathrm{Ph} . \mathrm{D}$. degree in Harbin Institute of Technology. His research interests include active noise control, adaptive signal processing, and sensor technology. 


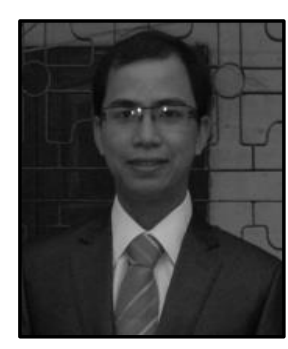

Van Nhu Le, he was born in 1982. He received the B.S degrees from the Le Quy Don Technical University, Hanoi, Vietnam, in 2007 and M.S degree from Harbin Institute of Technology in 2012. Now he is pursuing Ph.D. degree in Harbin Institute of Technology, Harbin, China. He currently focuses image processing, optic image processing and design optic system. 